\title{
SATELLITE OPERATORS WITH DISTINCT ITERATES IN SMOOTH CONCORDANCE
}

\author{
ARUNIMA RAY \\ (Communicated by Martin Scharlemann)
}

\begin{abstract}
Each pattern $P$ in a solid torus gives a function $P: \mathcal{C} \rightarrow \mathcal{C}$ on the smooth knot concordance group, taking any knot $K$ to its satellite $P(K)$. We give examples of winding number one patterns $P$ and a class of knots $K$, such that the iterated satellites $P^{i}(K)$ are distinct in concordance, i.e. if $i \neq j \geq 0$, $P^{i}(K) \neq P^{j}(K)$. This implies that the operators $P^{i}$ give distinct functions on $\mathcal{C}$, providing further evidence for the (conjectured) fractal nature of $\mathcal{C}$. Our theorem also allows us to construct several sets of examples, such as infinite families of topologically slice knots that are distinct in smooth concordance, infinite families of 2-component links (with unknotted components and linking number one) which are not smoothly concordant to the positive Hopf link, and infinitely many prime knots which have the same Alexander polynomial as an $L$-space knot but are not themselves $L$-space knots.
\end{abstract}

\section{INTRODUCTION}

A knot is the image of a smooth embedding $S^{1} \hookrightarrow S^{3}$. The satellite construction is a well-known function on $\mathcal{K}$, the set of isotopy classes of knots. Briefly, a satellite operator or pattern $P$ is a knot in a solid torus and the satellite knot $P(K)$ is obtained by tying the solid torus into the knot $K$. An example of the satellite construction is shown in Figure 1 a more precise definition is given in Section 2.1.



$P$

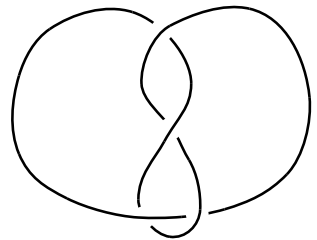

$K$

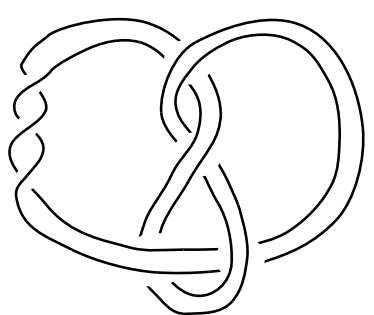

$P(K)$

Figure 1. The satellite construction on knots.

Two knots $K_{0} \hookrightarrow S^{3} \times\{0\}$ and $K_{1} \hookrightarrow S^{3} \times\{1\}$ are said to be concordant if they cobound a smooth, properly embedded annulus in $S^{3} \times[0,1] . \mathcal{K}$ modulo

Received by the editors April 21, 2014 and, in revised form, September 3, 2014.

2010 Mathematics Subject Classification. Primary 57M25.

The author was partially supported by NSF-DMS-1309081 and the Nettie S. Autrey Fellowship (Rice University). 


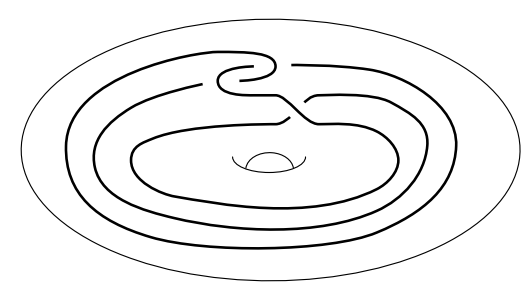

Figure 2. The Mazur operator $M$.

concordance forms an abelian group called the knot concordance group, denoted by $\mathcal{C}$. Similarly, we say that two knots are exotically concordant if they cobound a smooth, properly embedded annulus in a smooth 4 -manifold homeomorphic to $S^{3} \times[0,1]$ (but not necessarily diffeomorphic). $\mathcal{K}$ modulo exotic concordance forms an abelian group called the exotic knot concordance group, denoted by $\mathcal{C}^{\mathrm{ex}}$. If the 4-dimensional smooth Poincaré Conjecture is true, we can see that $\mathcal{C}=\mathcal{C}^{\text {ex }}[5$. The satellite operation on knots descends to well-defined functions on $\mathcal{C}$ and $\mathcal{C}^{\mathrm{ex}}$.

Satellite knots are interesting both within and without knot theory. Satellite operations can be used to construct distinct knot concordance classes which are hard to distinguish using classical invariants, such as in [10,11. In [6], winding number one satellite operators are used to construct non-concordant knots with homology cobordant zero-surgery manifolds. Satellite operations were used in [17] to subtly modify a 3-manifold without affecting its homology type. Winding number one satellite operators in particular are related to Mazur 4-manifolds [2] and Akbulut corks 1 .

There has been considerable interest in understanding the action of satellite operators on $\mathcal{C}$. For instance, it is a famous conjecture that the Whitehead double of a knot $K$ is smoothly slice if and only if $K$ is smoothly slice [22, Problem 1.38]. This question might be generalized to ask if operators are injective, that is, given an operator $P$, does $P(K)=P(J)$ imply $K=J$ in smooth concordance? A survey of some recent work on the Whitehead doubling operator may be found in [20]. In [10, several 'robust doubling operators' were introduced and evidence was provided for their injectivity. Not much else is known in the winding number zero case. For operators with non-zero winding numbers, there has been more success. Recently, Cochran, Davis and the author proved the following result.

Theorem 1 (Theorem 5.1 of [5]). If $P$ is a strong winding number one satellite operator, the induced function $P: \mathcal{C}^{e x} \rightarrow \mathcal{C}^{e x}$ is injective, i.e. for any two knots $K$ and $J, P(K)=P(J)$ if and only if $K=J$ in $\mathcal{C}^{e x}$. If the 4-dimensional smooth Poincaré Conjecture is true, $P: \mathcal{C} \rightarrow \mathcal{C}$ is injective.

The notion of a 'strong winding number one' satellite operator is described in Section 2.1. In particular, any winding number one operator which is unknotted as a knot in $S^{3}$ is strong winding number one; see Figure 2 for an example. Following A. Levine [24, we refer to the pattern in Figure 2 as the Mazur operator, or the Mazur pattern, and reserve the symbol $M$ for it.

Theorem 1 is related to the possibility of $\mathcal{C}$ having a fractal structure. This was conjectured in [10] where some evidence was provided to support this theory. One may characterize 'fractalness' of a set as the existence of self-similarities at arbitrarily small scales. By Theorem 1, any strong winding number one satellite operator 
gives a self-similarity for $\mathcal{C}^{\mathrm{ex}}$; however, while there exist several such operators (see [5. Section 2]), the question of scale has not been addressed. This is the objective of the main theorem of this paper, which follows.

Main Theorem. For any strong winding number one satellite operator $P$ with a Legendrian diagram where $\operatorname{tb}(P)>0$ and $\operatorname{tb}(P)+\operatorname{rot}(P) \geq 2$ (e.g. the Mazur pattern) and any knot $K$ with a Legendrian diagram realizing $\operatorname{tb}(K)=2 \mathrm{~g}(K)-1$, the knots $P^{i}(K)$ are distinct in $\mathcal{C}^{\text {ex }}$ and $\mathcal{C}$. That is, $P^{i}(K) \neq P^{j}(K)$ for all $i \neq j \geq 0$ in $\mathcal{C}$ and $\mathcal{C}^{e x}$.

In particular, for the Mazur pattern $M$ and knots $K$ as above,

$$
\begin{aligned}
\tau\left(M^{i}(K)\right)=\frac{\mathrm{s}\left(M^{i}(K)\right)}{2} & =\mathrm{g}_{4}\left(M^{i}(K)\right)=\mathrm{g}\left(M^{i}(K)\right) \\
& =\mathrm{g}(K)+i=\mathrm{g}_{4}(K)+i=\frac{\mathrm{s}(K)}{2}+i=\tau(K)+i .
\end{aligned}
$$

The action on $\mathcal{C}$ by these satellite operators should be compared to shrinking the Cantor ternary set by a factor of three, namely that each iteration gives a distinct image of $\mathcal{C}$ at smaller and smaller scales. To complete the fractal analogy one must also address the question of surjectivity of strong winding number one operators; some progress towards this end has been achieved by Davis and the author in 13 .

Examples of knots $K$ with the property that $\operatorname{tb}(K)=2 \mathrm{~g}(K)-1$ are plentiful. Any knot which is the closure of a positive braid (such as the positive torus knots) has this property. If a knot satisfies this condition, so does its untwisted Whitehead double [36]. Moreover, this property is preserved under connected sum of knots. Such knots have the additional property that $\mathrm{g}(K)=\mathrm{g}_{4}(K)=\tau(K)=\frac{\mathrm{s}(K)}{2}$; this is easily seen using the slice-Bennequin inequality (Proposition 2.2).

In addition to the Mazur pattern $M$, the main theorem applies to several other satellite operators. Two infinite families of such patterns are given in Figures 9 and [10. see Proposition 3.5 for exact calculations of various invariants for these families.

The question of whether the iterates of a satellite operator are distinct is an interesting question in its own right. In particular, there is no known counterpart of our main theorem for the Whitehead doubling operator. It was recently shown in [31] that for the torus knots $T_{2,2 m+1}$ with $m>2, \mathrm{Wh}\left(T_{2,2 m+1}\right)$ and $\mathrm{Wh}^{2}\left(T_{2,2 m+1}\right)$ are independent in $\mathcal{C}$; however, we are still unable to distinguish any of the other iterated Whitehead doubles of any knots. It is known that for knots $K$ with $\tau(K)>$ 0 , the iterated Whitehead doubles are all non-slice, but it is not known whether they are distinct (this result, of [18], was preceded by much other work on this topic, such as in [3, 7, 36]).

1.1. Applications of the main theorem. By a judicious choice of a knot $K$ and pattern $P$ for which our main theorem is applicable, we can ensure that each $P^{i}(K)$ will be topologically slice and $P^{i}(K) \neq P^{j}(K)$ for all $i \neq j \geq 0$ in smooth (as well as exotic) concordance. This gives us the following corollary.

Corollary 1. There exist infinite families of smooth (and exotic) concordance classes of topologically slice knots, where given any two knots in a family, one is a satellite of the other.

Several examples of infinite families of smooth concordance classes of topologically slice knots exist in the literature, such as in [14,16, 20, 21]. Our examples are 
novel only due to the ease with which they are obtained and the added property that they are iterated satellites.

We can also obtain an interesting corollary about $L$-space knots, which were defined in [30]. All positive torus knots, i.e. the knots $T_{p, q}$ with $p, q>0$, are well-known $L$-space knots. $L$-space knots have received much interest lately; in particular, they are relevant to the Berge conjecture (see 19 for background on the Berge conjecture and the role of $L$-space knots). The knot Floer complexes of $L$-space knots may be computed directly from their Alexander polynomials [30; it was also shown in [30] that there are strong restrictions on the Alexander polynomial of $L$-space knots. Using our main theorem, we obtain the following corollary.

Corollary 2. For any $L$-space knot $K$ with $\operatorname{tb}(K)>0$ (such as the positive torus knots), there exist infinitely many prime knots with the same Alexander polynomial as $K$ which are not themselves $L$-space knots.

As a third application of the main theorem, we can construct infinitely many links which are not smoothly concordant to the Hopf link. Any 2-component link $L=(P, \eta)$ with $\eta$ unknotted gives a satellite operator by considering the knot $P$ in the solid torus $S^{3}-N(\eta)$, where $N(\eta)$ is a regular neighborhood of $\eta$. It is easy to see that if two such links are concordant they give identical functions on $\mathcal{C}^{\text {ex }}$ [5. Proposition 2.3]. Given a satellite operator $P$, we may consider the associated 2-component link $(P, \eta(P))$ where $\eta(P)$ is the meridian of the solid torus containing $P$, and obtain the following corollary of our main theorem.

Corollary 3. For any operator $P$ in the main theorem, the associated links $\left(P^{i}\right.$, $\left.\eta\left(P^{i}\right)\right)$ yield distinct concordance classes of links with linking number one and unknotted components, which are each distinct from the class of the positive Hopf link.

By choosing $P$ carefully, we can obtain interesting families of links, without having to resort to too many direct calculations. In particular, we can obtain new links which are topologically concordant to the Hopf link but not smoothly so (the existence of such links was shown in [4]). These new examples will appear in a subsequent paper.

Recall that the $n$-solvable, positive, negative, and bipolar filtrations of $\mathcal{C}$ (from [11] and [9, denoted by $\left\{\mathcal{F}_{n}\right\},\left\{\mathcal{P}_{n}\right\},\left\{\mathcal{N}_{n}\right\}$, and $\left\{\mathcal{B}_{n}\right\}$ respectively) can be defined in terms of the zero-surgery manifolds of knots, i.e. whether a knot lies in a particular level of the filtration depends entirely on the homology cobordism class of its zero surgery manifold. Define the $n$-solvable height (resp. positive, negative, and bipolar height) of a knot $K$ to be the largest $k$ such that $K$ is in $\mathcal{F}_{k}$ (resp. $\mathcal{P}_{k}, \mathcal{N}_{k}$, and $\mathcal{B}_{k}$ ) or -1 if $K$ is in $\mathcal{C}-\mathcal{F}_{0}$ (resp. $\mathcal{C}-\mathcal{P}_{0}, \mathcal{C}-\mathcal{N}_{0}$, and $\left.\mathcal{C}-\mathcal{B}_{0}\right)$. We know from [6. Corollary 2.2] that if a winding number one satellite operator $P$ is slice (that is, $P(U)$ is slice, where $U$ is the unknot, e.g. the Mazur pattern), then the zero-surgery manifolds of $P(K)$ and $K$ are homology cobordant, for any knot $K$. As a result, we obtain the following corollary.

Corollary 4. For any knot $K$ with $n$-solvable (resp. positive, and bipolar) height $k$ and $\operatorname{tb}(K)=2 g(K)-1$, and a slice operator $P$ for which the main theorem applies, we obtain a family $\left\{P^{i}(K)\right\}$ of infinitely many distinct knots of $n$-solvable (resp. positive, and bipolar) height $k$.

Since if $K$ has $\operatorname{tb}(K)=2 \mathrm{~g}(K)-1$ it has $\tau(K)>0$ (and therefore negative height $-1)$, we cannot directly use the same construction for $\left\{\mathcal{N}_{n}\right\}$, but the mirror images 



FiguRE 3. The monoid operation on satellite operators.

of the examples for $\left\{\mathcal{P}_{n}\right\}$ suffice, i.e. suppose $K$ has $\operatorname{tb}(K)=2 \mathrm{~g}(K)-1$ and $-K$ has negative height $k$, then $\left\{-P^{i}(K)\right\}$ is an infinite family of knots with negative height $k$.

Remark. Shortly after the completion of this project, Adam Levine announced a complete calculation of $\tau(M(K))$ for the Mazur pattern $M$ (Figure 2) and any knot $K$ using bordered Floer homology [24]. This computation implies stronger results than ours in the case of the Mazur pattern, and also furthers the fractal analogy for the concordance group by showing that the Mazur pattern is non-surjective on $\mathcal{C}$; see [24] for more details. See also [8] for more on the fractal structure of $\mathcal{C}$ and $\mathcal{C}^{\text {ex }}$ as metric spaces.

\section{BACKGROUND}

2.1. Satellite operators. A satellite operator, or pattern, is a knot in the standard unknotted solid torus $V=S^{1} \times D^{2}$. The winding number of a satellite operator $P$, denoted by $w(P)$, is the signed count of the number of intersections of $P$ with a generic meridional disk of $V$.

The set of satellite operators is a monoid in the following natural way. Given a satellite operator $P$ in a solid torus $V$, we see the following curves:

- $\mu(P)$, the meridian of $P$ within $V$,

- $\lambda(P)$, the longitude of $P$ within $V$,

- $m(P)$, the meridian of $V$, and

- $\ell(P)$, the longitude of $V$.

Given operators $P, Q$ in solid tori $V(P), V(Q)$, we construct the composed pattern $P \star Q$ as follows. Let $N(Q)$ be a regular neighborhood of $Q$ inside $V(Q)$. Glue $V(Q)-N(Q)$ and $V(P)$ by identifying $\mu(Q) \sim m(P)$ and $\lambda(Q) \sim \ell(P)$. The resulting 3-manifold is a solid torus. The image of $P$ inside this solid torus is the desired operator $P \star Q$. An example of this construction is shown in Figure 3 ,

The well-known action of satellite operators on knots is closely related to the above construction. Given a knot $K$ and a satellite operator $P$ in a solid torus $V$, we obtain the satellite knot $P(K)$ as follows. Denote the meridian of $K$ by $\mu(K)$ and the longitude by $\lambda(K)$. Let $N(K)$ be a tubular neighborhood of $K$. Glue $S^{3}-N(K)$ and $V$ by identifying $\mu(K) \sim m(P)$ and $\lambda(K) \sim \ell(P)$. The resulting 3 -manifold is $S^{3}$ and the image of $P$ inside this manifold is the knot $P(K)$. An example of this construction is given in Figure 1. For a survey of the satellite construction see [25, p. 10] or [34, p. 111]. 
It is easily seen that $(P \star Q)(K)=P(Q(K))$, i.e. the satellite construction gives a monoid action on $\mathcal{K}$. We denote the satellite operator $P \star P \star \cdots \star P$ by $P^{i}$. Therefore, $P^{i}(K)=P(P(\cdots(K) \cdots))=(P \star P \star \cdots \star P) K$, i.e. we get the same result whether we start with a knot $K$ and apply $P$ to it $i$ times or we apply the composed pattern $P \star P \star \cdots \star P$ to $K$ once.

Given a satellite operator $P$, we denote by $\widetilde{P}$ the knot $P(U)$, where $U$ is the unknot. $P$ is said to be strong winding number one [5, Definition 1.1] if $m(P)$ normally generates $\pi_{1}\left(S^{3}-\widetilde{P}\right)$. If $\widetilde{P}$ is unknotted, $H_{1}\left(S^{3}-\widetilde{P}\right)$ and $\pi_{1}\left(S^{3}-\widetilde{P}\right)$ are canonically isomorphic and therefore an unknotted $P$ is strong winding number one if and only if it is winding number one [5, Proposition 2.1].

If the knots $K_{0}$ and $K_{1}$ are concordant in any 4 -manifold $M$ bounded by two disjoint copies of $S^{3}$, the satellites $P\left(K_{0}\right)$ and $P\left(K_{1}\right)$ are concordant in $M$ for every operator $P \subseteq V$. This is easily seen as follows. Let $C$ be the concordance between $K_{0}$ and $K_{1}$. We excise a neighborhood of $C$ and glue it in $V(P) \times[0,1]$. The image of $P \times[0,1]$ in the resulting manifold (which is diffeomorphic to $M$ ) is a concordance between $P\left(K_{0}\right)$ and $P\left(K_{1}\right)$. As a result, the satellite construction is well-defined on $\mathcal{C}$ and $\mathcal{C}^{\mathrm{ex}}$.

2.2. Legendrian knots and the slice-Bennequin inequality. An embedding of a knot $K$ in $S^{3}$ is said to be Legendrian if at each point in $S^{3}$ it is tangent to the 2 -planes of the standard contact structure on $S^{3}$. Legendrian knots can be studied concretely through their front projections as in Figure 4. Legendrian knots have two classical invariants, the Thurston-Bennequin number, $\operatorname{tb}(\cdot)$, and the rotation number, $\operatorname{rot}(\cdot)$, both of which can be easily calculated via front projections. See [15] for an excellent review of these and related concepts.

Given a Legendrian knot with positive Thurston-Bennequin number, we may repeatedly stabilize at the cost of increasing the rotation number to get a Legendrian diagram with zero Thurston-Bennequin number; such a diagram is a different Legendrian knot, but has the same topological realization as the original knot. See Figure 4 for an example. For a knot $K$, we will abuse notation and use the letter $K$ for any of its Legendrian realizations.

We will make use of the slice-Bennequin inequality [35], which states that for any Legendrian knot $K$,

$$
\operatorname{tb}(K)+|\operatorname{rot}(K)| \leq 2 \mathrm{~g}_{4}(K)-1 .
$$



$\operatorname{tb}(K)=1$



$\operatorname{tb}(K)=0$

Figure 4. Two different Legendrian realizations of the righthanded trefoil. 


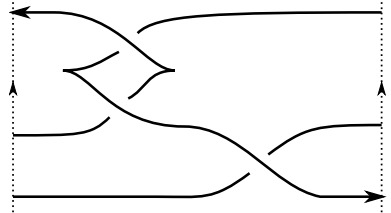

$\operatorname{tb}(M)=2, \operatorname{rot}(M)=0$

(a)

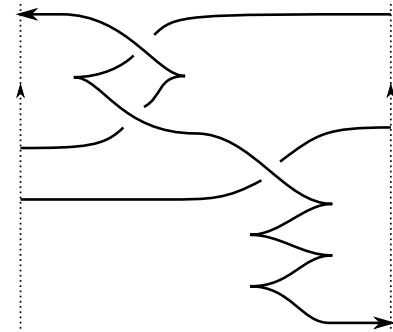

$\operatorname{tb}(M)=0, \operatorname{rot}(M)=2$

(b)

Figure 5. Two Legendrian fronts for the Mazur pattern $M$. The dashed vertical lines are identified to yield a knot in $S^{1} \times \mathbb{R}^{2}$ endowed with its natural contact structure obtained as a quotient of $\mathbb{R} \times \mathbb{R}^{2}$.

Here $\mathrm{g}_{4}(\cdot)$ stands for the smooth 4 -genus of a knot, i.e. the least genus of a connected, oriented, smooth, properly embedded surface bounded by $K$ in $B^{4}$. (There exist more general versions of this statement, due to [3, 26 and others. See [15, p. 133] for more details.) Since some of our work will be in the exotic category, we show that the slice-Bennequin inequality has an exotic analogue.

Definition 2.1. The exotic 4-genus of a knot $K$, denoted by $g_{4}^{\text {ex }}(K)$, is the least genus of a connected, oriented, smooth, properly embedded surface bounded by $K$ in a manifold $\mathcal{B}$ where $\partial \mathcal{B}=S^{3}$ and $\mathcal{B}$ is homeomorphic (but not necessarily diffeomorphic) to $B^{4}$.

Exotic 4-genus is clearly an invariant of exotic concordance of knots, and is bounded above by the (classical) smooth 4 -genus. The following is not original, but rather a collection of known results.

Proposition 2.2 (Exotic slice-Bennequin equality). For a Legendrian knot $K$ in $S^{3}$

$$
\begin{gathered}
\operatorname{tb}(K)+|\operatorname{rot}(K)| \leq s(K)-1 \leq 2 g_{4}^{e x}(K)-1 \leq 2 g_{4}(K)-1, \\
\operatorname{tb}(K)+|\operatorname{rot}(K)| \leq 2 \tau(K)-1 \leq 2 g_{4}^{e x}(K)-1 \leq 2 g_{4}(K)-1,
\end{gathered}
$$

where $s(K)$ is Rasmussen's invariant from Khovanov homology and $\tau(K)$ is Ozsváth-Szabó's invariant from Heegaard-Floer homology.

Proof. Corollary 1.1 of [23] shows that if $K$ bounds a connected, oriented, properly embedded surface $\Sigma$ in a homotopy 4 -ball $\mathcal{B}$, then $\mathrm{s}(K) \leq 2 \mathrm{~g}(\Sigma)$. Similarly, from Theorem 1.1 of [29, in the special case of homotopy 4-balls, $\tau(K) \leq \mathrm{g}(\Sigma)$. From 32,38 , we know that

$$
\operatorname{tb}(K)+|\operatorname{rot}(K)| \leq \mathrm{s}(K)-1
$$

and

$$
\operatorname{tb}(K)+|\operatorname{rot}(K)| \leq 2 \tau(K)-1,
$$

which completes the proof. 
2.3. The Legendrian satellite operation. The Legendrian satellite operation is discussed in [27,28]. There, a Legendrian pattern $P$ in $S^{1} \times \mathbb{R}^{2}$ acts on a Legendrian knot $K$ in $\mathbb{R}^{3}$. Front diagrams for the Mazur pattern is shown in Figure 5. (As in the case for Legendrian knots, we will abuse notation to use the symbol $P$ for all Legendrian realizations of a pattern $P$.) In order to construct the (Legendrian) satellite knot $P(K)$ we take an $n$-copy of $K$ ( $n$ 'vertical' parallels of $K$ ) and insert $P$ in a strand of $K$, where $n$ is the number of strands of $P$. This process is described in Figure 6. The resulting knot is the $\operatorname{tb}(K)$-twisted satellite of $K$ with pattern $P$. If $\operatorname{tb}(K)=0$, the resulting knot is a Legendrian realization of the classical untwisted satellite knot $P(K)$.

The same construction applies when a Legendrian pattern $P$ in $S^{1} \times \mathbb{R}^{2}$ acts on another pattern $Q$ also in $S^{1} \times \mathbb{R}^{2}$. This construction is described in Figure 7 , The resulting operator $P \cdot Q$ is the $\operatorname{tb}(Q)$-twisted $P$-satellite of $Q$. Therefore, if $\operatorname{tb}(Q)=0, P \cdot Q$ corresponds to the operator $P \star Q$ described in Section 2.1.

The following lemmata describe how the Thurston-Bennequin number and rotation number of satellites are related.

Lemma 2.3 (Remark 2.4 of [27]). For a Legendrian pattern $P$ with winding number $w(P)$ and a Legendrian knot $K$,

$$
\begin{aligned}
& \operatorname{tb}(P(K))=w(P)^{2} \operatorname{tb}(K)+\operatorname{tb}(P), \\
& \operatorname{rot}(P(K))=w(P) \operatorname{rot}(K)+\operatorname{rot}(P) .
\end{aligned}
$$

Lemma 2.4. For Legendrian patterns $P$ and $Q$ with winding number $w(P)$ and $w(Q)$ respectively,

$$
\begin{gathered}
\operatorname{tb}(P \cdot Q)=w(P)^{2} \operatorname{tb}(Q)+\operatorname{tb}(P), \\
\operatorname{rot}(P \cdot Q)=w(P) \operatorname{rot}(Q)+\operatorname{rot}(P) .
\end{gathered}
$$

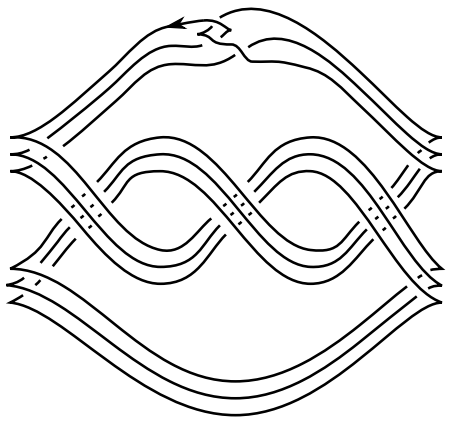

(a)

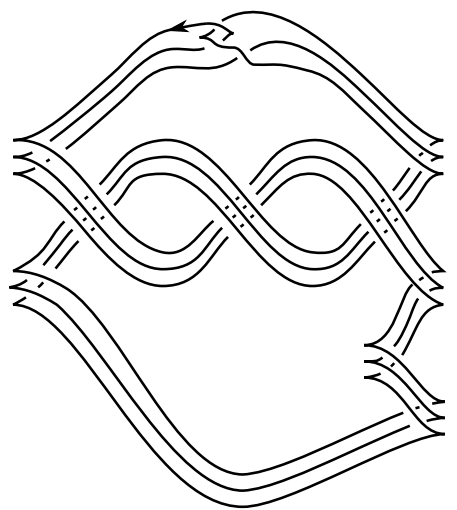

(b)

FiguRE 6 . The Legendrian satellite operation using $M$ in Figure 5(a) with the Legendrian fronts shown in Figure 4. The knot in (a) is therefore the 1-twisted classical satellite of $K$ with pattern $M$ and the knot in (b) is the (classical) untwisted satellite of $K$ with pattern $M$. 


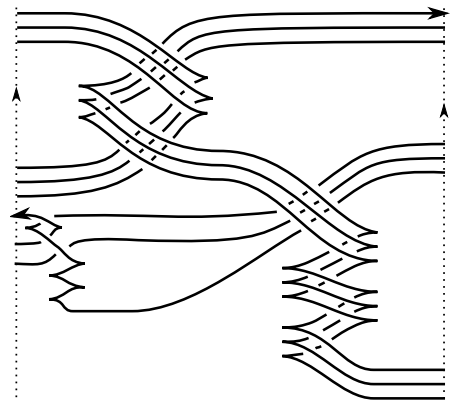

Figure 7. The pattern $M^{2}$, where $M$ is from Figure 5 (b).

Proof. As in Remark 2.4 of [27, these relationships are easily checked using front diagrams of $P$ and $Q$.

\section{Proof of the main theorem}

Proposition 3.1. For any strong winding number one satellite operator $P$ with a Legendrian diagram where $\operatorname{tb}(P)>0$ and $\operatorname{tb}(P)+\operatorname{rot}(P) \geq 2$ and non-slice knot $K$ with a Legendrian diagram realizing $\operatorname{tb}(K)=2 g(K)-1$ and $\operatorname{rot}(K)=0$, $P^{i}(K) \neq K$ for any $i>0$, even in exotic concordance.

Note that the following proof uses the technique of the proof of Theorem 3.1 in [6], which shows that $P(K) \neq K$.

Proof. Using positive stabilizations, we obtain a Legendrian diagram for $P$ with $\operatorname{tb}(P)=0$ and $\operatorname{rot}(P) \geq 2$, and a Legendrian diagram for $K$ with $\operatorname{tb}(K)=0$ and $\operatorname{rot}(K)=2 g(K)-1$.

Using formulae (2.1), (2.2), (2.3), and (2.4), we see that

$$
\begin{gathered}
\operatorname{tb}\left(P^{i}\right)=0, \\
\operatorname{rot}\left(P^{i}\right) \geq 2 i,
\end{gathered}
$$

and

$$
\begin{gathered}
\operatorname{tb}\left(P^{i}(K)\right)=0, \\
\operatorname{rot}\left(P^{i}(K)\right) \geq 2 \mathrm{~g}(K)-1+2 i,
\end{gathered}
$$

since $w\left(P^{i}\right)=1$. By the exotic slice-Bennequin equality, we have that

$$
0+|2 \mathrm{~g}(K)-1+2 i| \leq 2 \mathrm{~g}_{4}^{\mathrm{ex}}\left(P^{i}(K)\right)-1 .
$$

Note that $g_{4}^{\operatorname{ex}}(K) \leq \mathrm{g}(K)$ and $\mathrm{g}(K) \geq 1$. Therefore,

$$
g_{4}^{\mathrm{ex}}(K)+i \leq g_{4}^{\mathrm{ex}}\left(P^{i}(K)\right) .
$$

Therefore, for $i>0, K \neq P^{i}(K)$ even in exotic concordance.

Note that the above process also shows that

$$
\tau(K)+i \leq \tau\left(P^{i}(K)\right)
$$

and

$$
\mathrm{s}(K)+2 i \leq \mathrm{s}\left(P^{i}(K)\right)
$$

using the expanded versions of the exotic slice Bennequin inequality given in Proposition 2.2 and since $s(K) \leq 2 g_{4}^{\mathrm{ex}}(K)$ and $\tau(K) \leq g_{4}^{\mathrm{ex}}(K)$ for any knot $K$. 
Remark 3.2. In the particular case of the Mazur pattern $M$, there is an easier proof. We see that we can change $M^{i}(K)$ to $M^{i-1}(K)$ by changing a single positive crossing to a negative crossing. Therefore, by Corollary 1.5 of [29], we know that

$$
\tau\left(M^{i-1}(K)\right) \leq \tau\left(M^{i}(K)\right) \leq \tau\left(M^{i-1}(K)\right)+1
$$

and therefore,

$$
\tau(K) \leq \tau(M(K)) \leq \tau\left(M^{2}(K)\right) \leq \cdots \leq \tau\left(M^{i}(K)\right) .
$$

Recall that $\tau(\cdot)$ is an invariant of exotic concordance. Therefore, if $M^{i}(K)=K$ even in exotic concordance for some $i>0$, then $\tau\left(M^{i}(K)\right)=\tau(K)$. But this implies that for all $j$ with $0 \leq j \leq i, \tau\left(M^{j}(K)\right)=\tau(K)$. This contradicts Corollary 3.2 of [6], which shows exactly that $\tau(M(K))>\tau(K)$.

Proposition 3.3. For any strong winding number one satellite operator $P$ with a Legendrian diagram where $\operatorname{tb}(P)>0$ and $\operatorname{tb}(P)+\operatorname{rot}(P) \geq 2$ and non-slice knot $K$ with a Legendrian diagram realizing $\operatorname{tb}(K)=2 g(K)-1$ and $\operatorname{rot}(K)=0$, $P^{i}(K) \neq P^{j}(K)$ for any $i \neq j$, even in exotic concordance.

Proof. We know from Theorem 5.1 of 5 that $P$ is an injective operator, i.e. if $P(J)=P(K)$ in exotic concordance for any two knots $J$ and $K$, we can infer that $J=K$ in exotic concordance. Therefore, if $P^{i}(K)=P^{j}(K)$ for some $i>j$, we would have that $P^{i-j}(K)=K$ in exotic concordance, which contradicts Proposition 3.1.

For the Mazur operator $M$, we prove the following additional statements.

Proposition 3.4. For the Mazur operator $M$ and non-slice knot $K$ with a Legendrian diagram realizing $\operatorname{tb}(K)=2 g(K)-1$ and $\operatorname{rot}(K)=0$,

$$
\begin{gathered}
\tau\left(M^{i}(K)\right)=\tau(K)+i, \\
s\left(M^{i}(K)\right)=s(K)+2 i, \\
g_{4}^{e x}\left(M^{i}(K)\right)=g_{4}\left(M^{i}(K)\right)=g_{4}(K)+i, \\
g\left(M^{i}(K)\right)=g(K)+i,
\end{gathered}
$$

for all $i \geq 0$.

Recall that given such a $K, \mathrm{~g}(K)=\mathrm{g}_{4}(K)=\tau(K)=\frac{\mathrm{s}(K)}{2}$. Therefore, this proposition states that

$$
\begin{aligned}
\tau\left(M^{i}(K)\right) & =\frac{\mathrm{s}\left(M^{i}(K)\right)}{2}=\mathrm{g}_{4}^{\mathrm{ex}}\left(M^{i}(K)\right)=\mathrm{g}_{4}\left(M^{i}(K)\right)=\mathrm{g}\left(M^{i}(K)\right) \\
& =\mathrm{g}(K)+i=\mathrm{g}_{4}(K)+i=\mathrm{g}_{4}^{\mathrm{ex}}(K)+i=\frac{\mathrm{s}(K)}{2}+i=\tau(K)+i .
\end{aligned}
$$

Proof of Proposition 3.4. The first statement is a consequence of Corollary 1.5 of [29] as follows. We saw in the proof of Proposition 3.1 that $\tau(K)+i \leq \tau\left(M^{i}(K)\right)$. Since we can change $M^{i}(K)$ to $M^{i-1}(K)$ by changing a single positive crossing, by Corollary 1.5 of 29 , we have that

$$
\tau\left(M^{i}(K)\right) \leq \tau\left(M^{i-1}(K)\right)+1 \leq \cdots \leq \tau(K)+i .
$$

Therefore, $\tau\left(M^{i}(K)\right)=\tau(K)+i$.

Rasmussen's s-invariant behaves similarly under crossing changes, as shown in [33, Corollary 4.3]. Therefore,

$$
\mathrm{s}\left(M^{i}(K)\right) \leq \mathrm{s}\left(M^{i-1}(K)\right)+2 \leq \cdots \leq \mathrm{s}(K)+2 i
$$




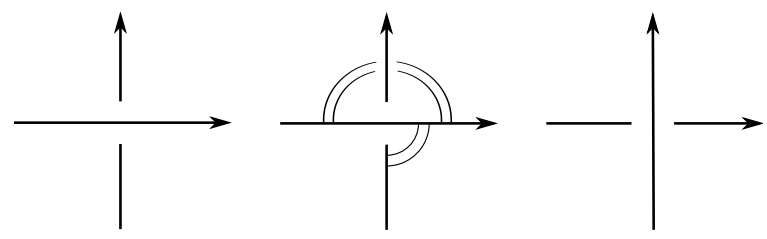

Figure 8. Two band additions can effect a crossing change.

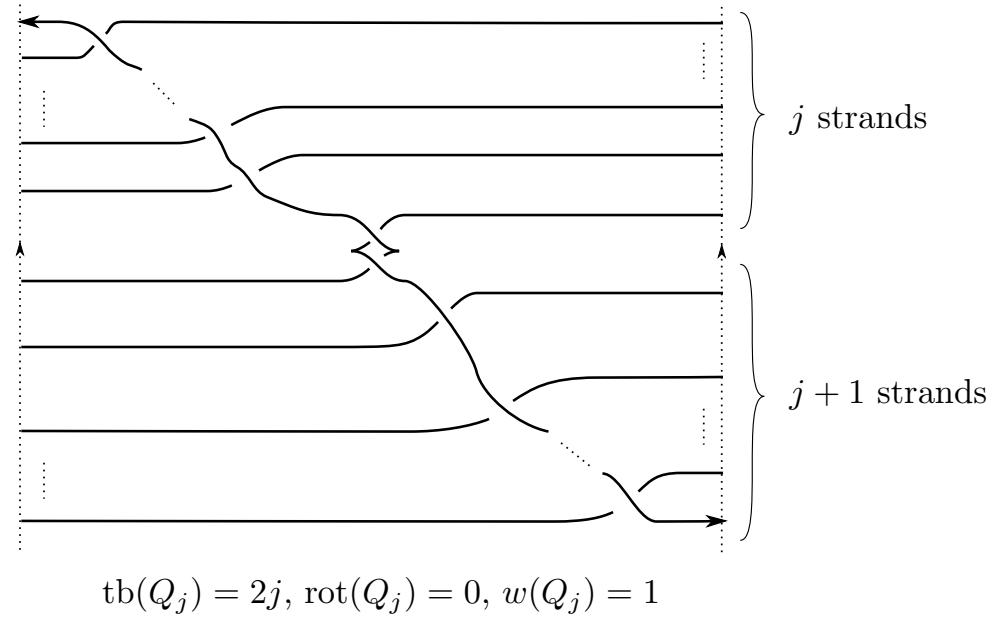

Figure 9. A Legendrian diagram for the winding number one pattern $Q_{j}$. Notice that $Q_{1}$ is the Mazur pattern.

and since we know from the proof of Proposition 3.1 that $\mathrm{s}(K)+2 i \leq \mathrm{s}\left(M^{i}(K)\right)$, we see that $\mathrm{s}\left(M^{i}(K)\right)=\mathrm{s}(K)+2 i$.

Similarly, we saw in the proof of Proposition 3.1 that $g_{4}(K)+i \leq g_{4}^{\text {ex }}\left(M^{i}(K)\right) \leq$ $g_{4}\left(M^{i}(K)\right)$. Since $M^{i}(K)$ and $K$ are related by a sequence of $i$ crossing changes each of which can be accomplished by adding two bands as shown in Figure 8 , we have that $g_{4}^{\mathrm{ex}}\left(M^{i}(K)\right) \leq g_{4}\left(M^{i}(K)\right) \leq g_{4}(K)+i$.

Since $\mathrm{g}(J) \geq \mathrm{g}_{4}(J)$ for any knot $J$, we must have that $\mathrm{g}\left(M^{i}(K)\right) \geq \mathrm{g}_{4}\left(M^{i}(K)\right)=$ $\mathrm{g}_{4}(K)+i$. Since $\mathrm{g}_{4}(K)=\mathrm{g}(K)$, we have that $\mathrm{g}\left(M^{i}(K)\right) \geq \mathrm{g}(K)+i$. One can construct a Seifert surface for $M^{i}(K)$ of genus $g(K)+i$, as pointed out in [6. Section $3]$. For $M(K)$, we can clearly see within the solid torus a genus one surface with two boundary components, one of which is the pattern $M$ and the other is the longitude of the solid torus. We glue this surface to a minimal genus Seifert surface for $K$, to see a Seifert surface for $M(K)$ with genus $g(K)+1$. Since $g_{4}(M(K))=g(P(K))$, we can proceed by induction.

The above computations can also be done for several other patterns. In particular, consider the families of patterns $Q_{j}$ and $R_{j}$ shown in Figure 9 and Figure 10 respectively. Note that the pattern $Q_{j}$ is changed to $Q_{j-1}$ by changing a single positive crossing at the clasp and $Q_{1}$ is the Mazur pattern $M$. Similarly, $R_{j}$ can be changed to $R_{j-1}$ by changing a single positive crossing at the top clasp, and $R_{0}$ is the identity operator (represented by the core of a solid torus). 


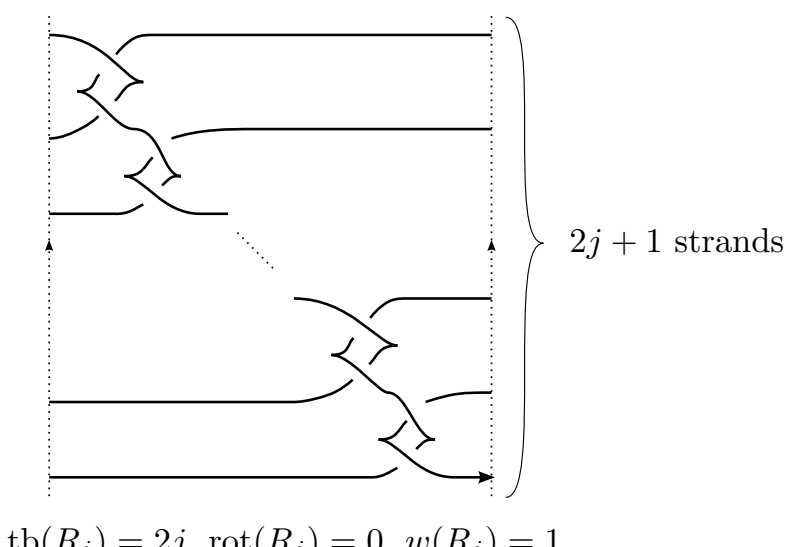

FIgURE 10. A Legendrian diagram for the winding number one pattern $R_{j}$. $R_{j}$ has $2 j$ clasps and $R_{0}$ is the identity satellite operator.

Proposition 3.5. For the patterns $Q_{j}$ and $R_{j}$ and any $j \geq 0$ (shown in Figures 9 and 10) and any non-slice knot $K$ with a Legendrian diagram realizing $\operatorname{tb}(K)=$ $2 g(K)-1$ and $\operatorname{rot}(K)=0$, we have that

$$
\begin{aligned}
\tau\left(Q_{j}(K)\right) & =\frac{s\left(Q_{j}(K)\right)}{2}=g_{4}^{e x}\left(Q_{j}(K)\right)=g_{4}\left(Q_{j}(K)\right)=g\left(Q_{j}(K)\right) \\
& =g(K)+j=g_{4}(K)+j=g_{4}^{e x}(K)+j=\frac{s(K)}{2}+i=\tau(K)+j, \\
\tau\left(R_{j}(K)\right) & =\frac{s\left(R_{j}(K)\right)}{2}=g_{4}^{e x}\left(R_{j}(K)\right)=g_{4}\left(R_{j}(K)\right)=g\left(R_{j}(K)\right) \\
& =g(K)+j=g_{4}(K)+j=g_{4}^{e x}(K)+j=\frac{s(K)}{2}+i=\tau(K)+j .
\end{aligned}
$$

For the iterated satellite operators $Q_{j}^{i}$ and $R_{j}^{i}$, for $i \geq 0$, we obtain the following:

$$
\begin{aligned}
\tau\left(Q_{j}^{i}(K)\right) & =\frac{s\left(Q_{j}^{i}(K)\right)}{2}=g_{4}^{e x}\left(Q_{j}^{i}(K)\right)=g_{4}\left(Q_{j}^{i}(K)\right)=g\left(Q_{j}^{i}(K)\right) \\
& =g(K)+i j=g_{4}(K)+i j=g_{4}^{e x}(K)+i j=\frac{s(K)}{2}+i j=\tau(K)+i j, \\
\tau\left(R_{j}^{i}(K)\right) & =\frac{s\left(R_{j}^{i}(K)\right)}{2}=g_{4}^{e x}\left(R_{j}^{i}(K)\right)=g_{4}\left(R_{j}^{i}(K)\right)=g\left(R_{j}^{i}(K)\right) \\
& =g(K)+i j=g_{4}(K)+i j=g_{4}^{e x}(K)+i j=\frac{s(K)}{2}+i j=\tau(K)+i j .
\end{aligned}
$$

We omit the proof of the above proposition since it is virtually identical to the proof of the main theorem. We see that the above statements yield the conditions we obtained in the proof of Proposition 3.4 for $Q_{1} \equiv M$, when we set $j=1$.

Together the results of this section constitute a proof of the main theorem. 


\section{Applications}

Corollary 1. There exist infinite families of smooth (and exotic) concordance classes $\left\{P^{i}(K)\right\}$ of topologically slice knots, where given any two knots in a family, one is a satellite of the other.

Proof. We can choose $K$ to be a topologically slice knot with a Legendrian realization such that $\operatorname{tb}(K)=2 \mathrm{~g}(K)-1$, such as the positive untwisted Whitehead double of any knot with this property [36. Choose a pattern $P$ such that $\widetilde{P}$ is topologically slice. If $K$ is topologically slice, then $P^{i}(K)$ is topologically concordant to $P^{i}(U)=\widetilde{P^{i}}$. Therefore, for any such knot $K$ and pattern $P$ we generate an infinite set of smooth concordance classes of topologically slice knots.

Corollary 2. For any $L-$ space knot $K$ with $\operatorname{tb}(K)>0$ (such as the positive torus knots), there exist infinitely many prime knots with the same Alexander polynomial as $K$ which are not themselves L-space knots.

Proof. For $L$-space knots $K$ which satisfy $\operatorname{tb}(K)=2 \mathrm{~g}(K)-1$ (such as the positive torus knots), this follows very easily from our main theorem. Recall that $M$ denotes the Mazur pattern. Note that $\Delta_{M^{i}(K)}(t)=\Delta_{\widetilde{M^{i}}}(t) \Delta_{K}(t)=\Delta_{K}(t)$ since $M$ is winding number one and $\widetilde{M}$ is unknotted [37, Theorem II]. However, for an $L-$ space knot $J, \tau(J)$ is equal to the degree of the symmetrized Alexander polynomial. Therefore, since each $M^{i}(K)$ has the same Alexander polynomial as $K$ but has distinct $\tau$-invariants (from the main theorem), they are not $L$-space knots. Since the patterns $M$ we use are unknotted as knots in $S^{3}$, the knots $M^{i}(K)$ are prime (see [12, Theorem 4.4.1]).

In the more general case for an $L$-space knot with $\operatorname{tb}(K)>0$, we can still stabilize the Legendrian diagram to get a diagram with $\operatorname{tb}((K))=0$ and rotation number $R$. Using the same techniques as in the proof of the main theorem, we obtain that

$$
2 i+|R| \leq 2 \tau\left(M^{i}(K)\right)-1 .
$$

This shows that $\left\{\tau\left(M^{i}(K)\right)\right\}_{i=0}^{\infty}$ is unbounded and monotone increasing, and that we can find a subsequence which is strictly increasing and bounded below by $\tau(K)$, which completes the proof.

Note that we can use these arguments for any other family of unknotted satellite operators as allowed by the main theorem as long as we can compute $\tau$-invariants, such as those shown in Figures 9 and 10 .

Corollary 3. For any operator $P$ in the main theorem, the associated links $\left(P^{i}\right.$, $\left.\eta\left(P^{i}\right)\right)$ yield distinct concordance classes of links with linking number one and unknotted components, which are each distinct from the class of the positive Hopf link.

Proof. By Proposition 7.1 of [5], since the functions $P^{i}$ give non-trivial functions on $\mathcal{C}^{\text {ex }}$, the corresponding links cannot be smoothly concordant to the positive Hopf link.

Corollary 4. For any knot $K$ with $n$-solvable (resp. positive, and bipolar) height $k$ and $\operatorname{tb}(K)=2 g(K)-1$, and a slice operator $P$ for which the main theorem applies, we obtain a family $\left\{P^{i}(K)\right\}$ of infinitely many distinct knots of $n$-solvable (resp. positive, and bipolar) height $k$. 
If a knot $K$ has $\operatorname{tb}(K)=2 g(K)-1$ and $-K$ has negative height $k$, then $\left\{-P^{i}(K)\right\}$ is an infinite family of knots with negative height $k$.

Proof. $n$-solvability (resp. positivity, negativity, and bipolarity) of a knot depends entirely on the homology cobordism class of its zero-surgery manifold; this is clearly seen from their definitions in [1] and [9]. The proof then follows immediately from the fact that if a winding number one satellite operator $P$ is slice (e.g. the Mazur pattern), then the zero-surgery manifolds of $P(K)$ and $K$ are homology cobordant, for any knot $K[6$, Corollary 2.2].

\section{ACKNOWLEDGEMENTS}

The author would like to thank Tim Cochran, Jung Hwan Park, and Christopher Davis for their time and insightful discussions. The author is also indebted to the participants of the Heegaard Floer "computationar" held at Rice University in Spring 2014, particularly Allison Moore and Eamonn Tweedy, for their insights into $L$-space knots and Heegaard-Floer homology. The comments from an anonymous referee greatly improved the exposition, particularly in Section 3 .

\section{REFERENCES}

[1] Selman Akbulut, A fake compact contractible 4-manifold, J. Differential Geom. 33 (1991), no. 2, 335-356. MR.1094459 (92b:57025)

[2] Selman Akbulut and Robion Kirby, Mazur manifolds, Michigan Math. J. 26 (1979), no. 3, 259-284. MR544597 (80h:57004)

[3] Selman Akbulut and Rostislav Matveyev, Exotic structures and adjunction inequality, Turkish J. Math. 21 (1997), no. 1, 47-53. MR1456158 (98d:57053)

[4] Jae Choon Cha, Taehee Kim, Daniel Ruberman, and Sašo Strle, Smooth concordance of links topologically concordant to the Hopf link, Bull. Lond. Math. Soc. 44 (2012), no. 3, 443-450, DOI 10.1112/blms/bdr103. MR2966989

[5] Tim D. Cochran, Christopher W. Davis, and Arunima Ray, Injectivity of satellite operators in knot concordance, J. Topol. 7 (2014), no. 4, 948-964. MR.3286894

[6] Tim D. Cochran, Bridget D. Franklin, Matthew Hedden, and Peter D. Horn, Knot concordance and homology cobordism, Proc. Amer. Math. Soc. 141 (2013), no. 6, 2193-2208, DOI 10.1090/S0002-9939-2013-11471-1. MR3034445

[7] Tim D. Cochran and Robert E. Gompf, Applications of Donaldson's theorems to classical knot concordance, homology 3-spheres and property $P$, Topology 27 (1988), no. 4, 495-512, DOI 10.1016/0040-9383(88)90028-6. MR976591 (90g:57020)

[8] Tim D. Cochran and Shelly Harvey, The geometry of the knot concordance space, Preprint, available at http://arxiv.org/abs/1404.5076, 2014.

[9] Tim D. Cochran, Shelly Harvey, and Peter Horn, Filtering smooth concordance classes of topologically slice knots, Geom. Topol. 17 (2013), no. 4, 2103-2162, DOI 10.2140/gt.2013.17.2103. MR.3109864

[10] Tim D. Cochran, Shelly Harvey, and Constance Leidy, Primary decomposition and the fractal nature of knot concordance, Math. Ann. 351 (2011), no. 2, 443-508, DOI 10.1007/s00208010-0604-5. MR2836668(2012k:57012)

[11] Tim D. Cochran, Kent E. Orr, and Peter Teichner, Structure in the classical knot concordance group, Comment. Math. Helv. 79 (2004), no. 1, 105-123, DOI 10.1007/s00014-001-0793-6. MR2031301 (2004k:57005)

[12] Peter R. Cromwell, Knots and links, Cambridge University Press, Cambridge, UK, 2004. MR2107964 (2005k:57011)

[13] Christopher W. Davis and Arunima Ray, Satellite operators as group actions on knot concordance, Preprint: http://arxiv.org/abs/1306.4632, 2013.

[14] Hisaaki Endo, Linear independence of topologically slice knots in the smooth cobordism group, Topology Appl. 63 (1995), no. 3, 257-262, DOI 10.1016/0166-8641(94)00062-8. MR1334309 (96c:57010) 
[15] John B. Etnyre, Legendrian and transversal knots, Handbook of knot theory, Elsevier B. V., Amsterdam, 2005, pp. 105-185, DOI 10.1016/B978-044451452-3/50004-6. MR2179261 (2006j:57050)

[16] Robert E. Gompf, Smooth concordance of topologically slice knots, Topology 25 (1986), no. 3, 353-373, DOI 10.1016/0040-9383(86)90049-2. MR842430(87i:57004)

[17] Shelly L. Harvey, Homology cobordism invariants and the Cochran-Orr-Teichner filtration of the link concordance group, Geom. Topol. 12 (2008), no. 1, 387-430, DOI 10.2140/gt.2008.12.387. MR.2390349(2009d:57045)

[18] Matthew Hedden, Knot Floer homology of Whitehead doubles, Geom. Topol. 11 (2007), 2277-2338, DOI 10.2140/gt.2007.11.2277. MR2372849(2008m:57030)

[19] Matthew Hedden, On Floer homology and the Berge conjecture on knots admitting lens space surgeries, Trans. Amer. Math. Soc. 363 (2011), no. 2, 949-968, DOI 10.1090/S00029947-2010-05117-7. MR2728591 (2012f:57006)

[20] Matthew Hedden and Paul Kirk, Instantons, concordance, and Whitehead doubling, J. Differential Geom. 91 (2012), no. 2, 281-319. MR2971290

[21] Jennifer Hom, The knot Floer complex and the smooth concordance group, Comment. Math. Helv. 89 (2014), no. 3, 537-570, DOI 10.4171/CMH/326. MR.3260841

[22] Rob Kirby ed., Problems in low-dimensional topology, Geometric topology (Athens, GA, 1993), AMS/IP Stud. Adv. Math., vol. 2, Amer. Math. Soc., Providence, RI, 1997, pp. 35473. MR 1470751

[23] P. B. Kronheimer and T. S. Mrowka, Gauge theory and Rasmussen's invariant, J. Topol. 6 (2013), no. 3, 659-674, DOI 10.1112/jtopol/jtt008. MR3100886

[24] Adam Simon Levine, Non-surjective satellite operators and piecewise-linear concordance, Preprint, available at http://arxiv.org/abs/1405.1125, 2014.

[25] W. B. Raymond Lickorish, An introduction to knot theory, Graduate Texts in Mathematics, vol. 175, Springer-Verlag, New York, NY, 1997. MR1472978 (98f:57015)

[26] P. Lisca and G. Matić, Stein 4-manifolds with boundary and contact structures, Topology Appl. 88 (1998), no. 1-2, 55-66, DOI 10.1016/S0166-8641(97)00198-3. Symplectic, contact and low-dimensional topology (Athens, GA, 1996). MR.1634563 (99f:57037)

[27] Lenhard L. Ng, The Legendrian satellite construction, Preprint: http://arxiv.org/abs/ 0112105, 2001.

[28] Lenhard Ng and Lisa Traynor, Legendrian solid-torus links, J. Symplectic Geom. 2 (2004), no. 3, 411-443. MR2131643(2005k:57051)

[29] Peter Ozsváth and Zoltán Szabó, Knot Floer homology and the four-ball genus, Geom. Topol. 7 (2003), 615-639, DOI 10.2140/gt.2003.7.615. MR.2026543 (2004i:57036)

[30] Peter Ozsváth and Zoltán Szabó, On knot Floer homology and lens space surgeries, Topology 44 (2005), no. 6, 1281-1300, DOI 10.1016/j.top.2005.05.001. MR2168576 (2006f:57034)

[31] Kyungbae Park, On independence of iterated whitehead doubles in the knot concordance group, Preprint: http://arxiv.org/abs/1311.2050, 2014.

[32] Olga Plamenevskaya, Bounds for the Thurston-Bennequin number from Floer homology, Algebr. Geom. Topol. 4 (2004), 399-406, DOI 10.2140/agt.2004.4.399. MR2077671 (2005d:57039)

[33] Jacob Rasmussen, Khovanov homology and the slice genus, Invent. Math. 182 (2010), no. 2, 419-447, DOI 10.1007/s00222-010-0275-6. MR2729272(2011k:57020)

[34] Dale Rolfsen, Knots and links, Mathematics Lecture Series, vol. 7, Publish or Perish, Inc., Houston, TX, 1990. Corrected reprint of the 1976 original. MR.1277811 (95c:57018)

[35] Lee Rudolph, Quasipositivity as an obstruction to sliceness, Bull. Amer. Math. Soc. (N.S.) 29 (1993), no. 1, 51-59, DOI 10.1090/S0273-0979-1993-00397-5. MR1193540 (94d:57028)

[36] Lee Rudolph, An obstruction to sliceness via contact geometry and "classical" gauge theory, Invent. Math. 119 (1995), no. 1, 155-163, DOI 10.1007/BF01245177. MR.1309974 (95k:57013)

[37] H. Seifert, On the homology invariants of knots, Quart. J. Math., Oxford Ser. (2) 1 (1950), 23-32. MR0035436 (11,735b) 
[38] Alexander N. Shumakovitch, Rasmussen invariant, slice-Bennequin inequality, and sliceness of knots, J. Knot Theory Ramifications 16 (2007), no. 10, 1403-1412, DOI 10.1142/S0218216507005889. MR2384833 (2008m:57034)

Department of Mathematics, MS-050, Brandeis University, 415 South St., Waltham, MASSACHusETtS 02453.

E-mail address: aruray@brandeis.edu 